\title{
Analysis of the B and Be-star populations of the double cluster $h$ and $\chi$ Persei
}

\author{
Amber N. Marsh, ${ }^{1}$ M. Virginia McSwain ${ }^{1}$ and Thayne Currie ${ }^{2}$ \\ ${ }^{1}$ Department of Physics, Lehigh University, Bethlehem, PA, USA \\ email: [anm506, mcswain] @lehigh.edu \\ ${ }^{2}$ Harvard Smithsonian Center for Astrophysics, Cambridge, MA, USA \\ email: tcurrie@cfa.harvard.edu
}

\begin{abstract}
We present blue optical spectra of 92 members of $h$ and $\chi$ Per obtained with the $W I Y N$ telescope at Kitt Peak National Observatory. From these spectra, several stellar parameters were measured for the B-type stars, including $v \sin i, T_{\text {eff }}, \log g_{\text {polar }}, M_{\star}$, and $R_{\star}$. Strömgren photometry was used to measure $T_{\text {eff }}$ and $\log g_{\text {polar }}$ for the Be stars. We also analyze photometric data of cluster members and discuss the near- to mid-infrared excesses of Be stars.
\end{abstract}

Keywords. open clusters and associations: individual (NGC 869, NGC 884), stars: emissionline, stars: Be

\section{Introduction}

NGC 869 and NGC 884 ( $h$ and $\chi$ Persei, respectively) comprise a well-known double cluster rich in massive B-type stars, and have been the focus of many studies over the years. Recent studies show that NGC 869 and NGC 884 have nearly identical ages of $\sim 13-14 \mathrm{Myr}$, common distance moduli of $\mathrm{d} M \sim 11.85 \mathrm{mag}$, and common reddenings of $E(B-V) \sim 0.5-0.55$ (Currie et al. 2009; Slesnick et al. 2002; Bragg \& Kenyon 2005).

Currie et al. (2008; hereafter C08) identified two populations of NGC 869 and NGC 884 stars with detected Spitzer MIPS $24 \mu \mathrm{m}$ excess emission: 20 A and F-type stars with luminous debris-disk emission and 57 brighter, earlier stars with weaker excess emission. They identify most of the latter group as candidate Be stars. However, only 21 were previously listed as Be stars (e.g., Bragg \& Kenyon 2002; Slesnick et al. 2002).

In this study, we analyze blue optical spectra of 92 early-type cluster members, including 16 candidate Be stars from C08, and investigate their near- to mid-infrared (IR) excesses. With continued monitoring of these stars in both the optical and IR regimes, we hope to explore these excesses as a reasonable means for identifying potential Be stars within clusters, as well as to investigate the transient natures of the disks surrounding the known Be stars in NGC 869 and NGC 884.

\section{Overview}

Blue optical spectra of 92 members of NGC 869 and NGC 884 were obtained on 2005 November 14-15 using the WIYN 3.5m telescope with the Hydra spectrograph. The observed spectra cover a wavelength range of 4250-4900 ̊.

Shown in Figure 1 are samples of the model spectral fits used to measure values for $v \sin i, T_{\text {eff }}$, and $\log g$ for B-type stars. $v \sin i$ was determined by comparing the HeI $\lambda \lambda 4387$, 4471,4713, and MgII $\lambda 4481$ lines with the Kurucz ATLAs9 models (Kurucz 1994) and taking a weighted average of these four values. For stars having $T_{\text {eff }} \geqslant 15000 \mathrm{~K}$, the TLUSTY BSTAR2006 models (Lanz \& Hubeny 2007) were used to find $T_{\text {eff }}$ and $\log g$ 
using the $\mathrm{H} \gamma$ line. For stars having $T_{\text {eff }} \leqslant 15000 \mathrm{~K}$, the Kurucz ATLAs9 models were used (Kurucz 1994). The method of Huang \& Gies (2006; hereafter HG06) was used to determine $\log g_{\text {polar }}$. For Be stars, Strömgren photometry available from the WEBDA database was used to derive $T_{\text {eff }}$ and $\log g_{\text {polar }}$ based on the methods of McSwain et al. (2008). The masses and radii for all stars were determined from the Schaller et al. (1992) evolutionary tracks, which are shown plotted with $T_{\text {eff }}$ and $\log g_{\text {polar }}$ in Figure 2.
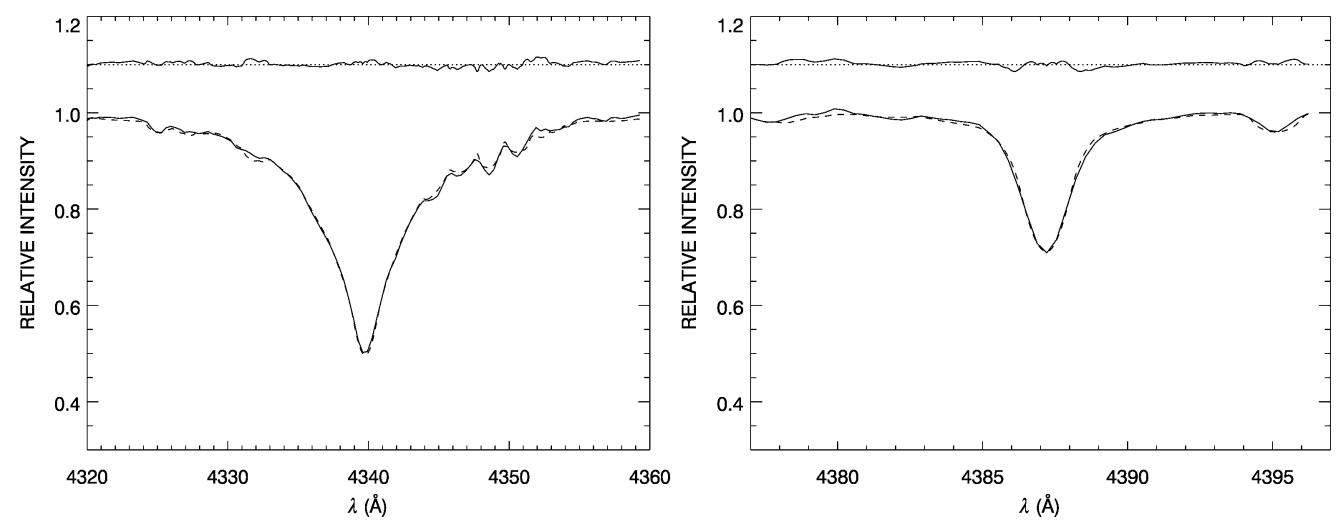

Figure 1. Sample spectral-line fits for NGC 869-90. Shown on the left is $\mathrm{H} \gamma$ and on the right is HeI $\lambda 4378$. The solid line is our observed spectrum while the dashed line displays our model fit to the line, with the computed residual shown above, shifted for clarity.
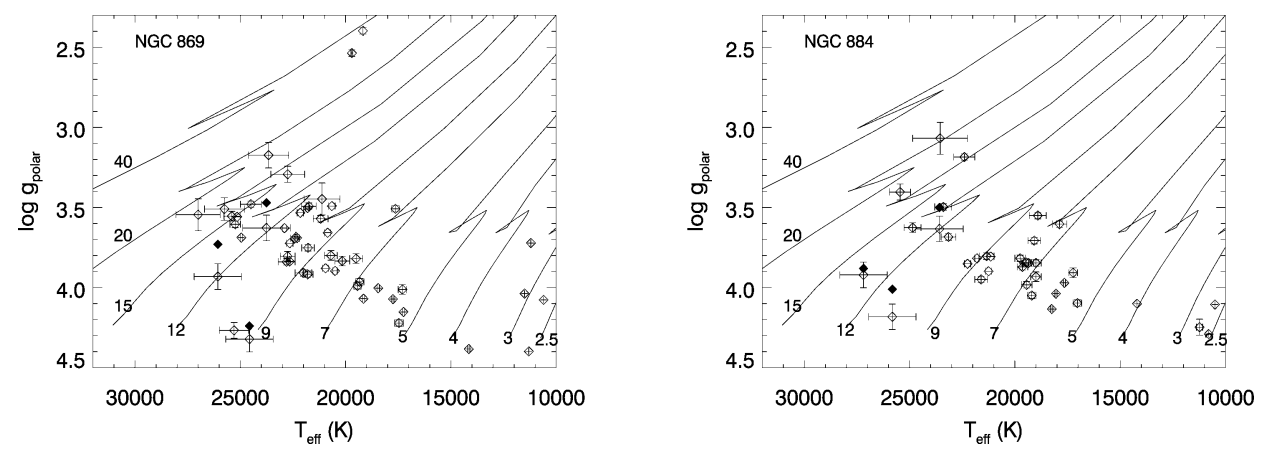

Figure 2. For both NGC 869 (left) and NGC 884 (right), $T_{\text {eff }}$ and $\log g_{\text {polar }}$ are plotted with the evolutionary tracks of Schaller et al. (1992). The zero-age main-sequence mass of each evolutionary track is labeled along the bottom. Normal B-type stars are shown as open diamonds while Be stars are filled diamonds.

\section{Results}

Sixteen Be candidates from C08 are present in our sample or that of HG06. Three of these 16 stars show no evidence of circumstellar emission in our spectra, although all have been observed to be Be stars in the past (Keller et al. 2001). Ten of the C08 Be candidates in our spectra show emission. Stellar parameters for the remaining three candidates are found in HG06, thus we cannot comment on the presence of emission at the time of observation. In addition, we find Be emission in one star (No. 1772) that was not observed by C08, and we present results for one additional star (No. 1268) identified as a Be star by Keller et al. (2001). These results are summarized in Table 1. 
Table 1. Measured physical parameters for Be stars

\begin{tabular}{|c|c|c|c|c|c|c|c|c|}
\hline $\begin{array}{l}\text { Cluster } \\
\text { Star }{ }^{1}\end{array}$ & $\begin{array}{c}v \sin i \\
\left(\mathrm{~km} \mathrm{~s}^{-1}\right)\end{array}$ & $\begin{array}{l}T_{\text {eff }} \\
(\mathrm{K})\end{array}$ & $\begin{array}{l}\log g_{\text {polar }} \\
\quad(\text { dex })\end{array}$ & $\begin{array}{c}M_{\star} \\
\left(\mathrm{M}_{\odot}\right)\end{array}$ & $\begin{array}{c}R_{\star} \\
\left(\mathrm{R}_{\odot}\right)\end{array}$ & $\begin{array}{c}\text { Be } \\
\text { Cand. }^{2}\end{array}$ & Comment & Ref. \\
\hline NGC $869-49$ & 172 & 23757 & 3.63 & 12.3 & 8.9 & Y & Emission present & This work \\
\hline NGC $869-517$ & 178 & $\ldots$ & $\ldots$ & $\ldots$ & $\ldots$ & Y & Emission present & This work \\
\hline NGC $869-566$ & 306 & 21183 & 3.57 & 10.4 & 8.7 & Y & No emission present ${ }^{3}$ & This work \\
\hline NGC $869-846$ & 205 & 22747 & 3.29 & 14.7 & 14.3 & Y & Weak emission present & This work \\
\hline NGC $869-847$ & 87 & 27000 & 3.54 & 17.8 & 11.8 & Y & Weak emission present & This work \\
\hline NGC 869-1162 & 66 & 19175 & 2.40 & 33.6 & 60.8 & $\mathrm{Y}$ & No emission present ${ }^{3}$ & This work \\
\hline NGC $869-1261$ & 285 & 26065 & 3.93 & 12.0 & 6.2 & $\mathrm{Y}$ & Strong emission present & This work \\
\hline NGC $869-1268$ & 151 & 24491 & 3.48 & 14.8 & 11.6 & $\mathrm{~N}$ & No emission present ${ }^{3,4}$ & This work \\
\hline NGC $869-1278$ & 197 & 24562 & 4.32 & 9.0 & 3.4 & Y & Emission present & This work \\
\hline NGC $884-1772$ & 379 & $\ldots$ & $\ldots$ & $\ldots$ & $\ldots$ & & Emission present & This work \\
\hline NGC $884-1926$ & 106 & 27190 & 3.92 & 13.5 & 6.7 & Y & Strong emission present & This work \\
\hline NGC 884-2091 & 236 & & & & $\cdots$ & Y & Emission present & This work \\
\hline NGC $884-2138$ & 153 & 23579 & 3.63 & 12.0 & 8.7 & Y & Emission present & This work \\
\hline NGC $884-2165$ & 79 & 26571 & 4.03 & 11.9 & 5.5 & Y & - & HG06 \\
\hline NGC $884-2402$ & 141 & 28238 & 3.81 & 15.6 & 8.1 & $\mathrm{Y}$ & - & HG06 \\
\hline NGC $884-2468$ & 134 & 10500 & 4.11 & 2.7 & 2.4 & $\mathrm{Y}$ & No emission present ${ }^{3}$ & This work \\
\hline NGC $884-2563$ & 308 & 25820 & 4.18 & 10.7 & 4.4 & Y & Strong emission present & This work \\
\hline NGC $884-2949$ & 168 & 18240 & 3.96 & 6.4 & 4.4 & Y & - & HG06 \\
\hline
\end{tabular}

Notes:

${ }^{1}$ Identification numbers from the WEBDA database. ${ }^{2}$ Be candidate in $\mathrm{C} 08 .{ }^{3}$ Stars not showing emission in our observations are likely transient Be stars. ${ }^{4}$ Identified as Be star by Keller et al. (2001).

Spectral-energy distributions (SEDs) for three stars in NGC 869 and NGC 884 are displayed in Figure 3. $U B V$ magnitudes are from the WEBDA database, $J H K_{\mathrm{s}}$ are from the 2MAss survey, and Spitzer [8] and [24] $\mu \mathrm{m}$ are from C08. These magnitudes were converted to fluxes using the methods detailed in Bessell et al. (1998), Cohen et al. (2003), Colina et al. (1996), Reach et al. (2005), and Rieke et al. (2008). Assuming a constant $E(B-V)=0.52 \mathrm{mag}$ for NGC 869 and NGC 884 (Bragg \& Kenyon 2005; Slesnick et al. 2002), reddened blackbody curves have been overlaid on these plots to investigate their near- to mid-IR excesses. All three stars shown in Figure 3 are proposed Be candidates (C08), with NGC 884-2138 and NGC 869-49 having emission present in our optical spectra and observed near- to mid-IR excess. NGC 884-2165 is not included in our spectroscopic sample but has previously been identified as a Be star and has observed IR excess (Keller et al. 2001).

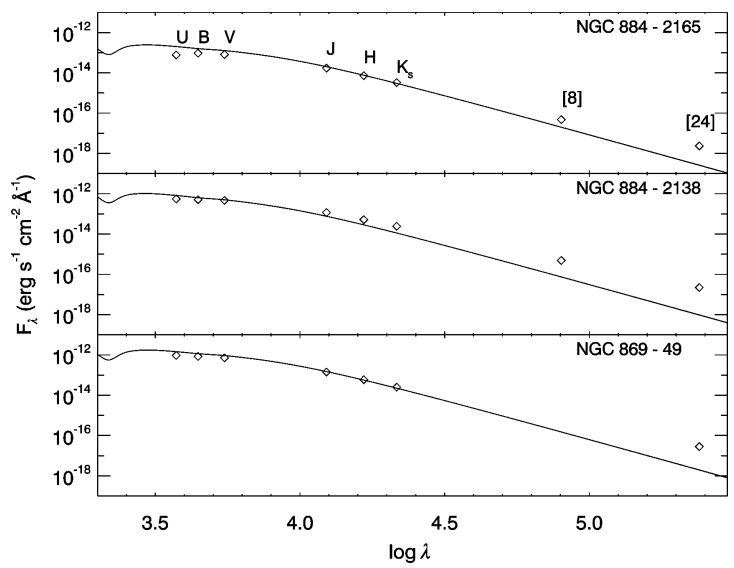

Figure 3. SEDs for three stars in NGC 869 and NGC 884. Reddened blackbody curves are overlaid on these plots to investigate their near- to mid-IR excesses. 


\section{Conclusions and further work}

We have measured the physical parameters of 77 B-type stars and 15 Be stars in NGC 896 and NGC 884. Sixteen Be candidates from C08 are present in our sample or that of HG06. Of these 16 Be candidates, three stars show no evidence of emission in our optical data and are likely transient Be stars. Ten of these Be candidates show emission in our spectra. Those Be candidates without emission in our spectra should be monitored in the future to further investigate their transient nature.

In the future, IRAC 3.6-5.8 $\mu \mathrm{m}$ data will be combined with the optical and IR fluxes used here to investigate the observed SEDs. We will fit the new SEDs using modern flux models rather than blackbody curves. Modifications accounting for variable reddening throughout the clusters will also be made. These new SED fits can then be used to model the Be disk sizes and temperatures.

\section{Acknowledgements}

We are grateful for travel support provided by the American Astronomical Society and the International Astronomical Union. We would like to thank Yale University for providing access to the WIYN telescope at KPNO. Institutional support was provided by Lehigh University. This work was also supported by NASA DPR No. NNX08AV70G.

\section{References}

Bessell, M. S., Castelli, F., \& Plez, B. 1998, A\&A, 333, 231

Bragg, A. \& Kenyon, S. 2002, AJ, 124, 3289

Bragg, A. \& Kenyon, S. 2005, AJ, 130, 134

Cohen, M., Wheaton, W. A., \& Megeath, S. T. 2003, AJ, 126, 1090

Colina, L., Bohlin, R. C., \& Castelli, F. 1996, AJ, 112, 307

Currie, T., Evans, N. R., Spitzbart, B. D., Irwin, J., Wolk, S. J., Hernandes, J., Kenyon, S. J., \& Pasachoff, J. M. 2009, AJ, 137, 3210

Currie, T., Kenyon, S. J., Balog, Z., Rieke, G., Bragg, A., \& Bromley, B. 2008, ApJ, 672, 558

Huang, W. \& Gies, D. R. 2006, ApJ, 648, 591

Keller, S. C., Grebel, E. K., Miller, G. J., \& Yoss, K. M. 2001, AJ, 122, 248

Kurucz, R. L. 1994, Kurucz CD-ROM 19, Solar Abundance Model Atmospheres for 0, 1, 2, 4, $8 \mathrm{~km} \mathrm{~s}^{-1}$, Cambridge: Smithsonian Astrophysical Observatory

Lanz, T. \& Hubeny, I. 2007, ApJS, 169, 83

McSwain, M. V., Huang, W., Gies, D. R., Grundstrom, E. D., \& Townsend, R. H. D. 2008, ApJ, 672,590

Reach, W. T., Megeath, S. T., Cohen, M., Hora, J., Carey, S., Surace, J., Willner, S. P., Barmby, P., Wilson, G., Glaccum, W., Lowrance, P., Marengo, M., \& Fazio, G. G. 2005, PASP, 117, 978

Rieke, G. H., Blaylock, M., Decin, L., Engelbracht, C., Ogle, P., Avrett, E., Carpenter, J., Cutri, R. M., Armus, L., Gordon, K., Gray, R. O., Hinz, J., Su, K., \& Willmer, C. N. A. 2008, AJ, 135,2245

Schaller, G., Schaerer, D., Meynet, G., \& Maeder, A. 1992, A\&\&AS, 96, 269

Slesnick, C., Hillenbrand, L. A., \& Massey, P. 2002, ApJ, 576, 880 